\title{
Bone marrow-derived mesenchymal stem cells modified with IGFBP-3 inhibit the proliferation of pulmonary artery smooth muscle cells
}

\author{
GE SHENG CHENG，YU SHUN ZHANG，TING TING ZHANG，LU HE and XING YE WANG \\ Department of Cardiovascular Medicine, The First Affiliated Hospital of \\ Xi'an Jiaotong University, Xi'an, Shaanxi 710061, P.R. China
}

Received November 28, 2015; Accepted November 10, 2016

DOI: $10.3892 / \mathrm{ijmm} .2016 .2820$

\begin{abstract}
Pulmonary arterial hypertension (PAH) is a common clinical cardiovascular disease, leading to the excessive proliferation of pulmonary artery smooth muscle cells (PASMCs) and endothelial cells, and is associated with a high mortality rate. Recently, stem- and progenitor cell-mediated gene therapies have provided a novel approach for the treatment of PAH. However, the function of human bone marrow-derived mesenchymal stem cells (hBM-MSCs) modified with the insulin-like growth factor binding protein-3 (IGFBP-3) gene in the regulation of PAH is not yet fully understood. In this study, we explored the biological role of IGFBP-3-modified hBM-MSCs in the proliferation of human PASMCs (hPASMCs), and also investigated the potential underlying molecular mechanisms. Our results revealed that $I G F B P-3$-modified hBM-MSCs inhibited the proliferation of angiotensin II-stimulated hPASMCs following co-culture on cell culture inserts. In addition, total DNA synthesis and the protein levels of hPASMCs in co-culture were decreased. Moreover, the IGFBP-3-modified hBM-MSCs promoted apoptosis and downregulated the expression of B-cell lymphoma-2 (Bcl-2), but increased the expression of Bcl-2 associated $X$ protein (Bax) in hPASMCs. Furthermore, the IGFBP-3-modified hBM-MSCs significantly induced a phenotypic change in the hPASMCs from the synthetic to the contractile phenotype in co-culture. Importantly, the
\end{abstract}

Correspondence to: Dr Ting Ting Zhang, Department of Cardiovascular Medicine, The First Affiliated Hospital of Xi'an Jiaotong University, 277 Yanta East Road, Xi'an, Shaanxi 710061, P.R. China

E-mail: cgs501@163.com

Abbreviations: $\mathrm{PAH}$, pulmonary arterial hypertension; hBM-MSCs, human bone marrow-derived mesenchymal stem cells; hPASMCs, human pulmonary artery smooth muscle cells; IGFBP-3, insulin-like growth factor binding protein-3

Key words: human bone marrow-derived mesenchymal stem cells, insulin-like growth factor binding protein-3, human pulmonary artery smooth muscle cells, cell proliferation, pulmonary arterial hypertension levels of several related proteins in the hPASMCs, including phosphorylated (p-)insulin receptor substrate-1 (p-IRS-1), phosphoinositide 3-kinase (p-PI3K), serine/threonine-protein kinase (p-Akt), mitogen-activated protein kinase (p-p38), p-Jun $\mathrm{N}$-terminal kinase (p-JNK) and extracellular signal-regulated kinase (p-ERK), were markedly decreased by the IGFBP-3modified hBM-MSCs following co-culture. Taken together, our findings suggest that IGFBP-3-modified hBM-MSCs inhibit the proliferation and promote the apoptosis of hPASMCs, and promote the swithc to a contractile phenotype in more effectively than wild-type hBM-MSCs, possibly through the activation of the PI3K/Akt and Ras-mitogen-activated protein kinase (MAPK) signaling pathways. The findings of our study suggest that IGFBP-3-modified hBM-MSCs may be a promising therapeutic strategy for the treatment of $\mathrm{PAH}$.

\section{Introduction}

Pulmonary arterial hypertension (PAH), whether idiopathic or of other varied etiologies, is a common clinical syndrome characterized by the sustained elevation of pulmonary vascular resistance, inflammatory cell infiltration, vascular remodeling and the occlusion of vessels with thrombi, leading to right ventricular hypertrophy or failure and, ultimately, death (1-4). Although the pharmacotherapy currently available for PAH can improve the quality of life of patients to a certain degree, the mortality rate remains high (5). Recently, mesenchymal stem cell (MSCs) and gene therapies have emerged as novel methods for the treatment of $\mathrm{PAH}$, including adipose tissue-derived MSCs, bone marrow-derived MSCs (BM-MSCs), hematopoietic stem cells and endothelial progenitor cells (6). MSCs not only have several favorable features, such as the ease of isolation, expansion in culture and their capacity to differentiate into multiple lineages, but they also migrate to sites of injury and generate prominent paracrine effects through key interactions with the immune system (7). Importantly, stem cell-mediated gene therapy appears to be more advantageous compared with sole stem cell therapy and gene therapy in PAH (8). Nevertheless, the mechanisms underlying their function are not yet completely clear and require further investigation.

The insulin-like growth factor binding protein (IGFBP) family inhibit cell proliferation, migration and survival and 
also play an important role in the stability of vascular remodeling $(9,10)$. IGFBP-3, a member of the IGFBP family, is a predominantly secreted protein (11) and has been shown to regulate the insulin-like growth factor (IGF) signaling pathway by restricting the access of IGFs to IGF receptors, consequently inhibiting their proliferative and anti-apoptotic actions at the extracellular level (12). In addition, IGFBP-3 inhibits growth and enhances apoptosis in an IGF-independent manner in several mammalian cells (13). In contrast to its anti-growth and apoptotic roles, IGFBP-3 promotes vascular regrowth and has cytoprotective properties in response to a range of cellular conditions (14). The ability of IGFBP-3 to pivot cell fate to either death or survival is associated with the cellular microenvironment, including the presence of IGFBP-3 binding partners and growth factor receptors (12).

The excessive proliferation of pulmonary artery smooth muscle cells (PASMCs) plays an essential role in the pathogenesis of vascular remodeling in PAH (15). It has been demonstrated that calcitonin gene-related peptide $(C G R P)$-modified MSCs secrete CGRP protein and inhibit the proliferation and migration of vascular smooth muscle cells (VSMCs) (16). It has also been demonstrated that IGFBP-3 expression is found in BM-MSCs and PASMCs isolated from PAH-afflicted rats (17), but its functional role in PASMCs remains unknown. Whether IGFBP-3 overexpression and human IGFBP-3-modified hBM-MSCs can suppress the proliferation of hPASMCs is also currently unknown. Therefore, in this study, we investigated the growth-inhibitory effects of IGFBP-3-modified hBM-MSCs on hPASMCs and also aimed to elucidate the underlying mechanisms.

\section{Materials and methods}

Cell culture. The hBM-MSCs (PCS-500-012 ${ }^{\mathrm{TM}}$ ) and hPASMCs (PCS-100-023 ${ }^{\mathrm{TM}}$ ) were purchased from the American Type Culture Collection (ATCC, Manassas, VA, USA). The hBM-MSCs used were clonally derived and the hPASMCs were used from passages 4 to 7. The cells were maintained in Dulbecco's modified Eagle's medium (DMEM) (Gibco, Grand Island, NY, USA) containing 10\% fetal bovine serum (FBS) (HyClone; Logan, Utah, USA), 1\% L-glutamine, $100 \mathrm{U} / \mathrm{ml}$ penicillin and $100 \mathrm{mg} / \mathrm{ml}$ streptomycin (Invitrogen, Burlington, ON, USA). Angiotensin II (Ang II) (Sigma-Aldrich, St. Louis, MO, USA) was used to stimulate hPASMC proliferation. These cells were grown in a humidified incubator with $5 \% \mathrm{CO}_{2}$ at $37^{\circ} \mathrm{C}$.

Construction of pcDNA4-IGFBP-3 vector and transfection of $h B M-M S C s$. The recombinant plasmid, pcDNA4-IGFBP-3, was constructed as previously described (19), verified and reproduced. The hBM-MSCs were seeded at $1 \times 10^{6}$ cells/ well in 6-well plates for $24 \mathrm{~h}$ and transfected with the pcDNA4-IGFBP-3 plasmid or pcDNA4 empty vector using Lipofectamine 2000 (Invitrogen, Carlsbad, CA, USA), according to the manufacturer's instructions. Following $48 \mathrm{~h}$ of transfection, the supernatants were harvested for enzymelinked immunosorbent assay (ELISA).

ELISA for IGFBP-3. Following $48 \mathrm{~h}$ of co-culture of the hBM-MSCs and hPASMCs on cell culture inserts, the supernatants of hPASMCs on the bottom chamber were collected and the production of IGFBP-3 was measured using the Quantikine human IGFBP-3 immunoassay (R\&D Systems, Minneapolis, $\mathrm{MN}, \mathrm{USA}$ ), according to the manufacturer's instructions.

Co-culture of hBM-MSCs and hPASMCs on cell culture inserts. An indirect co-culture system was assembled using Costar Transwell membranes (12 mm diameter, $0.4 \mu \mathrm{m}$ pore; Corning Inc., Corning, NY, USA). As described previously (20), the hPASMCs were first seeded on the bottom chamber in DMEM containing Ang II and hBM-MSCs transfected with pcDNA4-IGFBP-3 or pcDNA4 empty vector and then seeded on a $0.4 \mu \mathrm{m}$ Transwell membrane (upper chamber) and cultured in DMEM. Single cultures of hBM-MSCs or hPASMCs were used as controls. Co-cultures were maintained for $48 \mathrm{~h}$. This assay was performed at least 3 times.

$C C K-8$ assay. The proliferation of the hPASMC was evaluated by Cell Counting kit-8 (CCK-8) assay (Dojindo, Kumamoto, Japan) according to the manufacturer's instructions. A viability $>100 \%$ indicated cell proliferation, whereas a viability of $<100 \%$ indicated cell damage, as previously described (21). Briefly, the hPASMCs were plated in 96-well plates at 5,500 cells/well and $1.5 \mu \mathrm{M} / 1$ Ang II was added to each well. Following $48 \mathrm{~h}$ of incubation at $37^{\circ} \mathrm{C}, \mathrm{CCK}-8$ solution was added to each pore and incubation was continued for $3 \mathrm{~h}$. The absorbance value $(450 \mathrm{~nm})$ of each pore was analyzed using an enzyme symbolized meter (Bio-Rad Laboratories Inc., Hercules, CA, USA). This assay was performed at least 3 times.

Bromodeoxyuridine (BrdU) incorporation assay. The hBM-MSCs and hPASMCs were co-cultured for $48 \mathrm{~h}$ on cell culture inserts and the DNA synthesis ability of the hPASMCs was determined by a BrdU incorporation assay. Briefly, the hPASMCs were incubated with BrdU (Sigma) (20 $\mu \mathrm{l} /$ well) for $12 \mathrm{~h}$ and the Fixing/Denaturing solution (200 $\mu \mathrm{l} /$ well $)$ was then added followed by incubation for $30 \mathrm{~min}$. The BrdU detection antibody solution (200 $\mu \mathrm{l} /$ well) was then added and the cells were incubated for $1 \mathrm{~h}$. Subsequently, the peroxidase-labeled sheep anti-mouse IgG solution $(200 \mu \mathrm{l} /$ well $)$ was added followed by incubation for $30 \mathrm{~min}$. Finally, tetramethylbenzidine substrate $(100 \mu \mathrm{l})$ was added followed by incubation for $30 \mathrm{~min}$ in the dark. The amount of BrdU incorporation into the cells was measured at $450 \mathrm{~nm}$ using a microplate reader (Bio-Rad) according to the manufacturer's instructions. The experiments were performed in quintuplicate and repeated 3 times.

Flow cytometric analysis of apoptosis. Following co-culture of the hBM-MSCs and hPASMCs on cell culture inserts for $48 \mathrm{~h}$, the apoptosis of the hPASMCs was detected by BD Annexin V fluorescein isothiocyanate (FITC)/propidium iodide (PI) staining (BD Biosciences, San Jose, CA, USA) according to the manufacturer's instructions. As previously described (22), the hPASMCs were harvested, washed with icecold phosphate-buffered saline (PBS), resuspended in binding buffer and stained with $10 \mu \mathrm{l}$ of FITC Annexin V buffer at room temperature in the dark for $15 \mathrm{~min}$. To that, $5 \mu \mathrm{l}$ of PI was added followed by incubation for a further $5 \mathrm{~min}$. Finally, the cells were analyzed using a flow cytometer (BD Biosciences, Franklin Lakes, NJ, USA). The percentage of cell numbers in each quadrant was calculated using BD CellQuest software. 

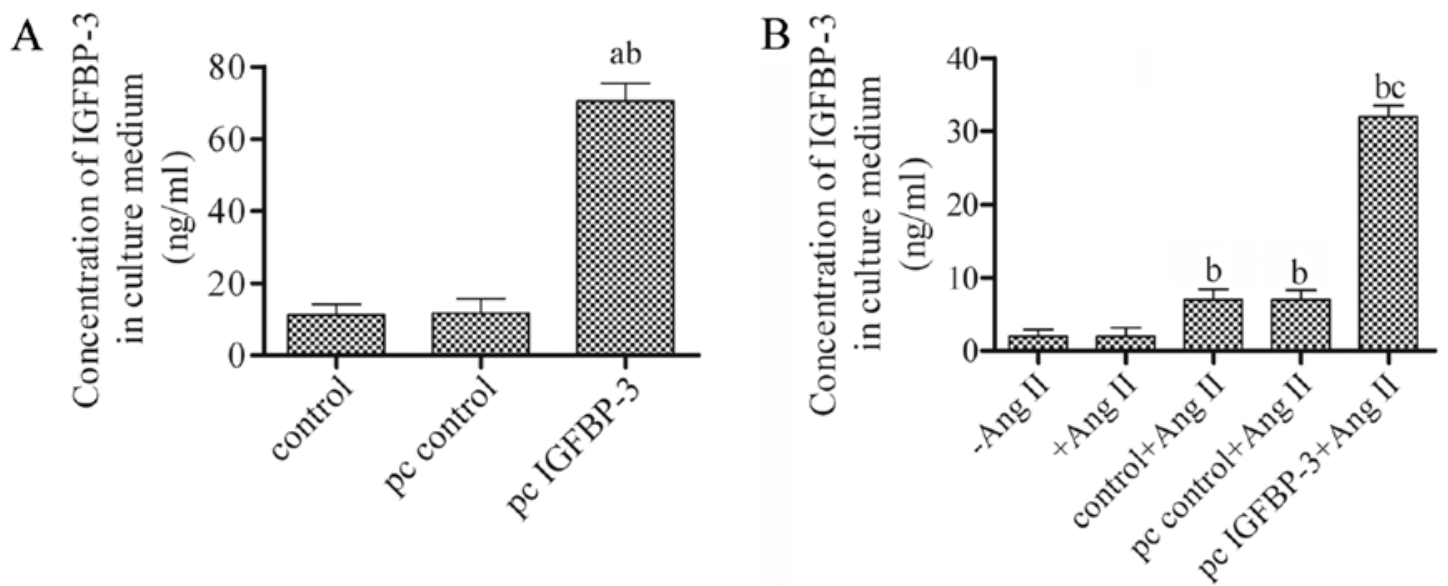

Figure 1. Overexpression and secretion of insulin-like growth factor binding protein-3 (IGFBP-3) in human bone marrow-derived mesenchymal stem cells (hBM-MSCs). (A) The supernatant was harvested after culturing the hBM-MSCs for $48 \mathrm{~h}$ and the concentration of IGFBP-3 (ng/ml) was detected by ELISA. (B) Co-culture of hBM-MSCs with human pulmonary artery smooth muscle cells (hPASMCs) for $48 \mathrm{~h}$ and the concentration of IGFBP-3 in the culture medium was detected by ELISA. ${ }^{\mathrm{a}} \mathrm{P}<0.05$ as compared with control or $-\mathrm{Ang}$ II; ${ }^{\mathrm{b}} \mathrm{P}<0.05$ vs. +Ang II; ${ }^{\mathrm{C}} \mathrm{P}<0.05$ vs. pc control + Ang II. Ang II, angiotensin II; -Ang II, untreated hPASMCs; +Ang II, Ang II-stimulated hPASMCs; control + Ang II, Ang II-stimulated hPASMCs not transfected with any plasmid; pc control + Ang II, Ang II-stimulated hPASMCs transfected with empty vector; pc IGFBP-3 + Ang II, Ang II-stimulated hPASMCs transfected with IGFBP-3 expression plasmid.

Protein extraction and western blot analysis. Total protein from the hPASMCs was extracted using RIPA lysis buffer containing phenylmethylsulfonyl fluoride and using the bicinchoninic acid (BCA) protein assay kit (Boster Biology Co., Wuhan, China), the protein concentration was then determined with BSA as the standard. Equal amounts of protein were separated on sodium dodecyl sulfate (SDS)-polyacrylamide gels and electrotransferred onto polyvinylidene fluoride membranes (Bio-Rad), followed by incubation at $4^{\circ} \mathrm{C}$ overnight with primary antibodies: anti- $\alpha$-smooth muscle-actin ( $\alpha$-SM-actin; sc-32251), anti-osteopontin (OPN; sc-21742), anti-B-cell lymphoma-2 (Bcl-2; sc-492), anti-Bax (sc-493), anti-insulin receptor substrate-1 (IRS-1; sc-559), anti-phosphoinositide 3-kinase (PI3K; sc-1637), anti-AKT (sc-1618), anti-p38 (sc-535), anti-p-Jun N-terminal kinase (JNK; sc-7345), anti-extracellular signal-regulated kinase (ERK; sc-292838) (Santa Cruz Biotechnology, Santa Cruz, CA, USA); anti-p-IRS-1 (\#2381), anti-p-PI3K (\#4228), anti-p-AKT (\#12694), anti-p-p38 (\#9211), anti-p-JNK (\#9251), anti-p-ERK (\#4376) (Cell Signaling Technology, Inc., Beverly, MA, USA). The appropriate secondary antibody: anti-mouse and anti-rabbit IgG horseradish peroxidase-linked antibody (Cell Signaling Technology, Inc.), was applied at room temperature for $1 \mathrm{~h}$. Finally, the protein band of interest was visualized by a chemiluminescent reaction using an ECL Detection kit (Pierce Biotechnology, Rockford, IL, USA). Bands were quantified using Image Lab $^{\mathrm{TM}}$ software, version 5.1 (Bio-Rad).

Reversetranscription-quantitative (real-time) $P C R(R T-q P C R)$. Total RNA was extracted from the hPASMCs using TRIzol reagent(Invitrogen) according tothe manufacturer'sinstructions and cDNA was synthesized using PrimeScript RT Master Mix Sample kit (Takara, Shiga, Japan) according to the manufacturer's instructions. Subsequently, qPCR was performed using SYBR-Green Master Mix and analyzed on a LightCycler 480 instrument (Roche Diagnostics Ltd., Lewes, UK). The oligonucleotide primers used for RT-qPCR were as follows: $\alpha$-SMA forward, 5'-CGGGACATCAAGGAGAAACT-3' and reverse, 5'-CCCATCAGGCAACTCGTAA-3'; OPN forward, 5'-GCCAGTTGCAGCCTTCTCA-3' and reverse, 5'-AAAAGCAAATCACTGCAATTCTCA-3'. All real-time reactions were performed using 50 cycles at $95^{\circ} \mathrm{C}$ for $10 \mathrm{~min}$, $95^{\circ} \mathrm{C}$ for $15 \mathrm{sec}$ and $60^{\circ} \mathrm{C}$ for $1 \mathrm{~min}$. Each sample was run in triplicate and $\beta$-actin served as an internal control. Relative mRNA expression was normalized to that of $\beta$-actin using the equation of $2^{-\Delta \Delta C T}$, where $\mathrm{CT}$ is threshold cycle. RT-qPCR was performed at least 3 times.

Statistical analysis. In this study, the results were summarized as the means $\pm \mathrm{SD}$ from at least 3 independent experiments. SPSS 17.0 software was used to carry out statistical analyses. The analysis of the overall effects of the different treatments was evaluated using one-way analysis of variance (ANOVA). A value of $\mathrm{P}<0.05$ was considered to indicate a statistically significant difference.

\section{Results}

Overexpression and secretion of IGFBP-3 in hBM-MSCs. To investigate the role of IGFBP-3 in hBM-MSCs, pcDNA4-IGFBP-3 or pcDNA4 empty vector was constructed and transfected into the hBM-MSCs. The supernatant was harvested after $48 \mathrm{~h}$ and the levels of secreted IGFBP-3 were then measured by ELISA. The results revealed a generally low concentration of IGFBP-3 either in the untreated hBM-MSCs (control) or in those transfected with the pcDNA4 empty vector (pc control). However, the concentration of IGFBP-3 was significantly increased when the hBM-MSCs were transfected with pcDNA4IGFBP-3 (pc IGFBP-3) (Fig. 1A). These data indicated that the hBM-MSCs transfected with pcDNA4-IGFBP-3 had a high secretion level of IGFBP-3 and support the notion that IGFBP-3 is secreted in the supernatant of MSCs (18). Moreover, the concentration of IGFBP-3 in the culture medium significantly increased compared to the control + Ang II or pc control + Ang II group, following co-culture with hBM-MSCs transfected with pcDNA4-IGFBP-3 and Ang II-stimulated hPASMCs on cell culture inserts (Fig. 1B). 

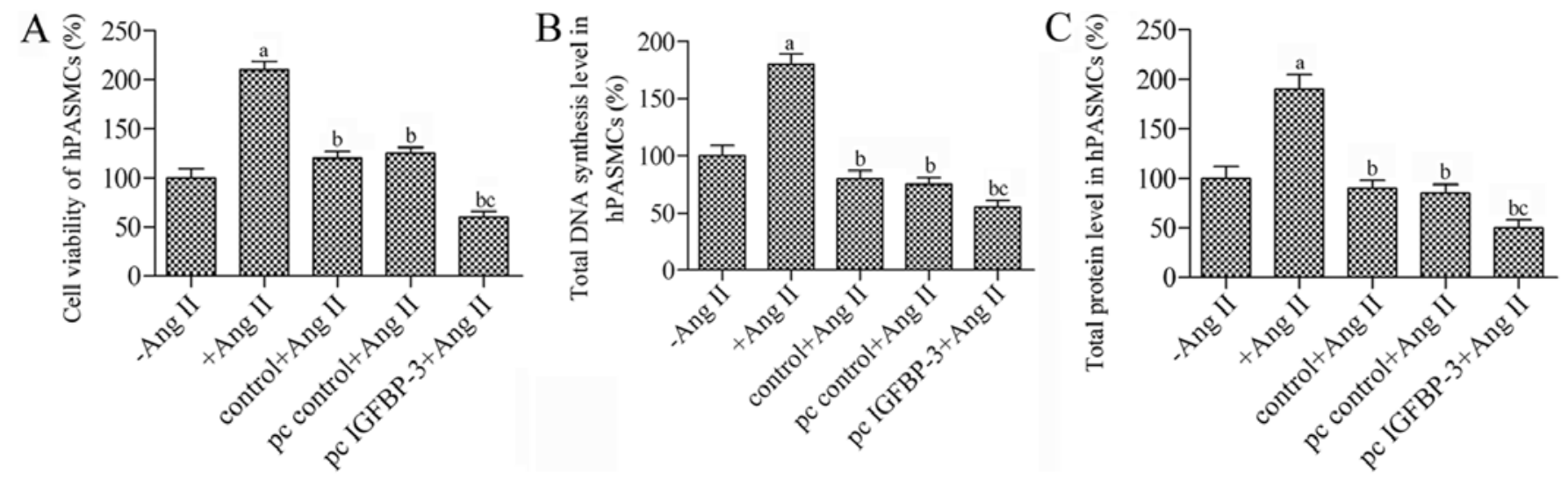

Figure 2. Effect of human bone-derived marrow mesenchymal stem cells (hBM-MSCs) on proliferation, DNA synthesis and total protein levels in human pulmonary artery smooth muscle cells (hPASMCs) stimulated with angiotensin II (Ang II). (A) Cell proliferation was assessed by CCK-8 assay. (B) DNA synthesis was examined by BrdU incorporation. (C) Total protein expression was detected by the BCA method. ${ }^{\mathrm{a}} \mathrm{P}<0.05$ vs. $-\mathrm{Ang}$ II; ${ }^{\mathrm{b}} \mathrm{P}<0.05$ vs. $+\mathrm{Ang}$ II; ${ }^{\mathrm{c}} \mathrm{P}<0.05$ vs pc control + Ang II. -Ang II, untreated hPASMCs; +Ang II, Ang II-stimulated hPASMCs; control + Ang II, Ang II-stimulated hPASMCs not transfected with any plasmid; pc control + Ang II, Ang II-stimulated hPASMCs transfected with empty vector; pc IGFBP-3 + Ang II, Ang II-stimulated hPASMCs transfected with IGFBP-3 expression plasmid.

hBM-MSCs modified with IGFBP-3 inhibit the proliferation of hPASMCs stimulated with Ang II. In order to determine whether IGFBP-3 overexpression in hBM-MSCs suppresses the proliferation of hPASMCs, an indirect co-culture system for hBM-MSCs and hPASMCs was used to observe the proliferation of the hPASMCs. Ang II resulted in a 2.1-fold increase in the proliferation of hPASMCs ( $\mathrm{P}<0.05$ vs. control) (Fig. $2 \mathrm{~A}$ ). Co-culture of the untreated hBM-MSCs decreased the survival rate of the hPASMCs by $80 \%$ in response to Ang II, while co-culture with the IGFBP-3-modified hBM-MSCs significantly inhibited the proliferation of the Ang II-stimulated hPASMCs; we observed a decrease of 1.33-fold compared to the pc control $(\mathrm{P}<0.05)$. Furthermore, DNA synthesis and the total protein levels in the hPASMCs in co-culture with the IGFBP-3-modified hBM-MSCs were decreased almost to $55 \%(\mathrm{P}<0.05)$ (Fig. 2B and $\mathrm{C})$. Taken together, these results suggest that co-culture with IGFBP-3-modified hBM-MSCs is an effective strategy to suppress the proliferation of hPASMCs.

Effects of the upregulation of IGFBP-3 on $\alpha$-SM-actin and $O P N$ expression in hPASMCs. The hPASMCs can modulate their phenotype from a contractile to a synthetic one under certain conditions. To examine the phenotypic switch of hPASMCs co-cultured with hBM-MSCs, the $\alpha$-SM-actin and OPN expression levels were examined by western blot analysis and RT-qPCR. The results revealed that the hBM-MSCs transfected with pcDNA4-IGFBP-3 had significantly increased protein and mRNA expression levels of $\alpha$-SM-actin in the hPASMCs $(\mathrm{P}<0.05)$ (Fig. 3A and B). Conversely, OPN protein and mRNA expression decreased by approximately $50 \%$ in the hPASMCs co-cultured with the IGFBP-3-modified hBM-MSCs $(\mathrm{P}<0.05)$ when compared with the untreated hBM-MSCs (Fig. 3C and D). These results suggest that the hPASMCs underwent a change in phenotype from a synthetic to a contractile phenotype following co-culture with IGFBP-3modified hBM-MSCs.

hBM-MSCs modified with IGFBP-3 promote the apoptosis of hPASMCs. To further investigate the effect of IGFBP-3- modified hBM-MSCs on hPASMCs, we detected cell apoptosis using the Annexin V FITC/PI assay, and Bax and Bcl-2 protein expression were examined by western blot analysis. Following co-culture of the hPASMCs stimulated with Ang II with the IGFBP-3-modified hBM-MSCs for $48 \mathrm{~h}$, the apoptosis of the hPASMC increased to $50 \%$ (Fig. 4A and B) compared with the pc control + Ang II group. The Bcl-2 expression levels were decreased, while Bax expression was increased in the hPASMCs co-cultured with the IGFBP-3-modified hBM-MSCs (Fig. 4C and D). Taken together, these results suggest that co-culture with the IGFBP-3-modified hBM-MSCs promotes the apoptosis of hPASMCs.

Effects of the overexpression of IGFBP-3 on the PI3K-AKT and mitogen-activated protein kinase (MAPK) pathways in hPASMCs. To determine the underlying mechanisms through which the IGFBP-3-modified hBM-MSCs regulate hPASMCs, we examined IRS-1 expression and the expression of related proteins of two signaling pathways, PI3K-AKT and MAPK, including PI3K, AKT, p38, JNK and ERK by western blot analysis. The results revealed that co-culture with the $I G F B P-3$-modified hBM-MSCs significantly attenuated the activities of the related proteins compared to those of the wildtype hBM-MSCs (pc control + Ang II) (Fig. 5). These data indicated that the IGFBP-3-modified hBM-MSCs markedly downregulated the expression of related genes in the PI3K-AKT and MAPK pathways in hPASMCs.

\section{Discussion}

The results of this study are the first to demonstrate that IGFBP3-modified hBM-MSCs significantly inhibit the proliferation and promote the apoptosis of hPASMCs stimulated with Ang II. We also found that the hPASMCs underwent a phenotypic transformation from a synthetic to a contractile phenotupe when in co-culture with $I G F B P$-3-modified hBM-MSCs. This study suggests that IGFBP-3-modified hBM-MSCs downregulate P13K-AKT and MAPK signaling pathways in hPASMCs more effectively than wild-type hBM-MSCs. 

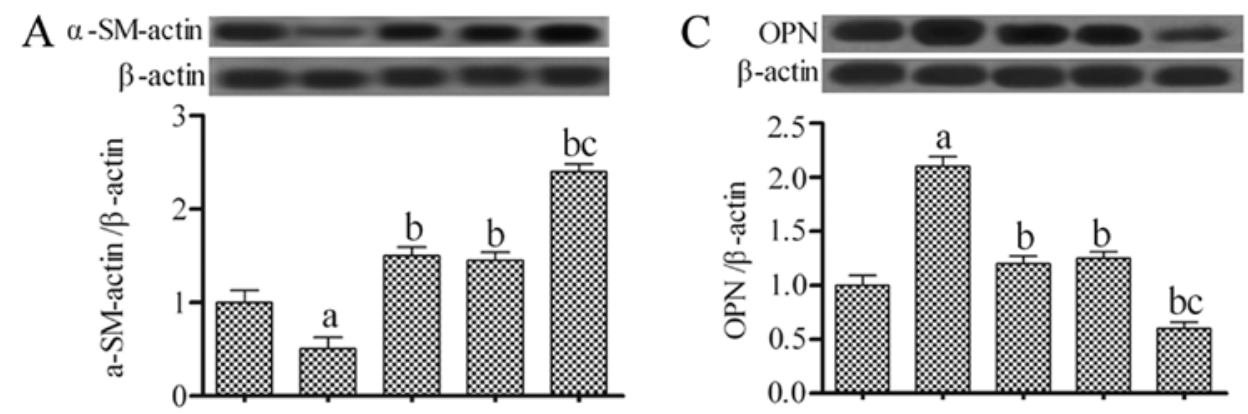

$\mathrm{B}$

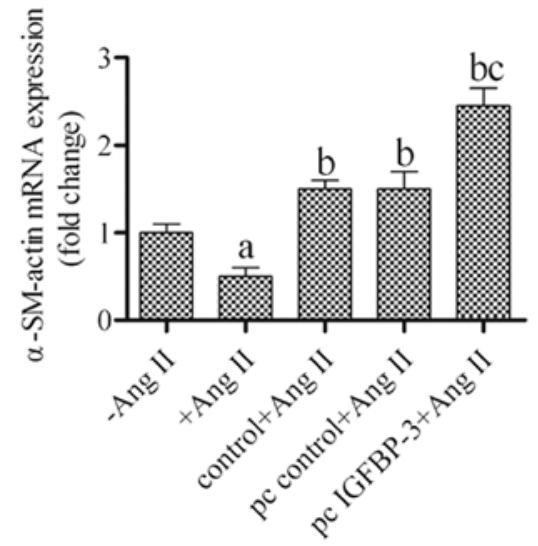

$\mathrm{D}$

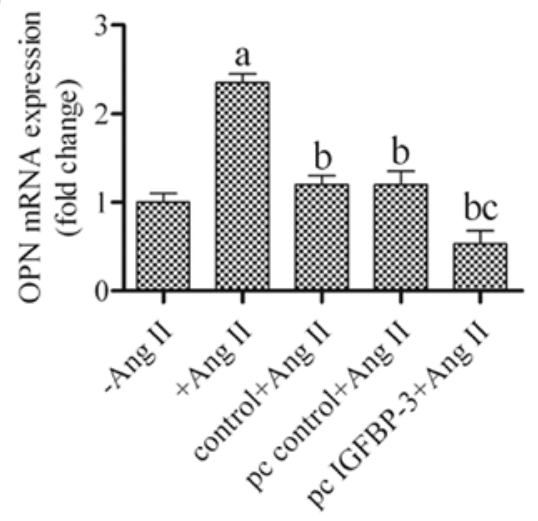

Figure 3. Effects of insulin-like growth factor binding protein-3 (IGFBP-3) upregulation on $\alpha$-smooth muscle-actin ( $\alpha$-SM-actin) and osteopontin (OPN) expression in human pulmonary artery smooth muscle cells (hPASMCs). (A and B) $\alpha$-SM-actin and (C and D) OPN expression assessed by western blot analysis and RT-qPCR. ${ }^{\mathrm{a}} \mathrm{P}<0.05$ vs. -Ang II; ${ }^{\mathrm{b}} \mathrm{P}<0.05$ vs. +Ang II; ${ }^{\mathrm{C}} \mathrm{P}<0.05$ vs. pc control + Ang II. Ang II, angiotensin II; -Ang II, untreated hPASMCs; +Ang II, Ang II-stimulated hPASMCs; control + Ang II, Ang II-stimulated hPASMCs not transfected with any plasmid; pc control + Ang II, Ang II-stimulated hPASMCs transfected with empty vector; pc IGFBP-3 + Ang II, Ang II-stimulated hPASMCs transfected with IGFBP-3 expression plasmid.

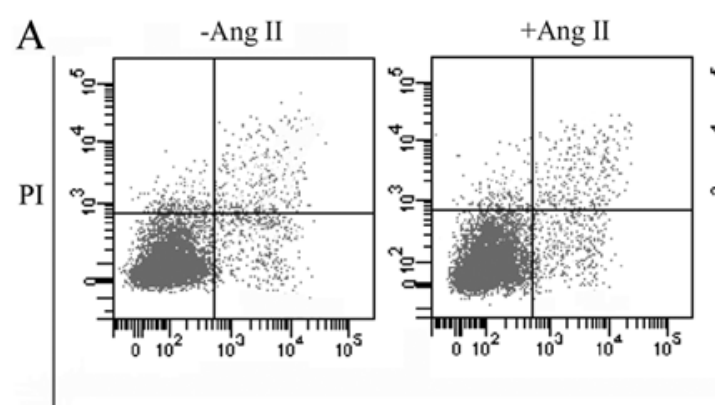

B

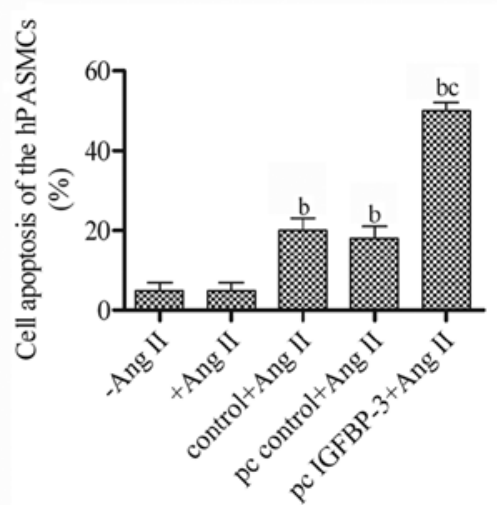

C $\quad$ Bct2 2 $\beta$-actin

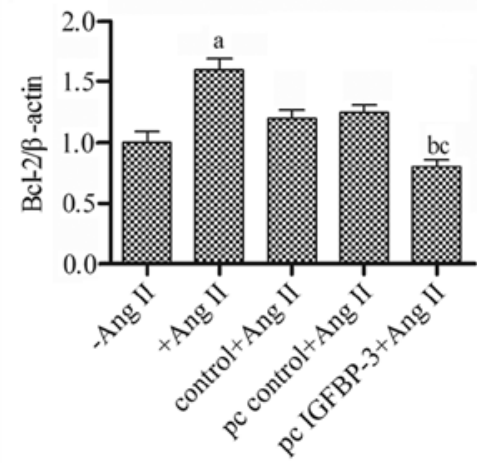

D $\operatorname{Bax}$ $\beta$-actin

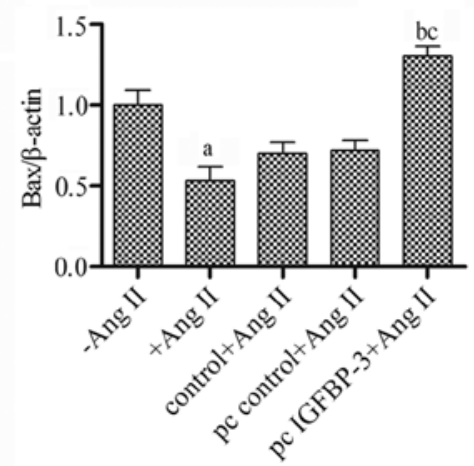

Figure 4. Upregulation of insulin-like growth factor binding protein-3 (IGFBP-3) in human bone marrow-derived mesenchymal stem cells (hBM-MSCs) promotes the apoptosis of human pulmonary artery smooth muscle cells (hPASMCs). (A) Apoptosis of hPASMC examined by flow cytometric analysis. (B) Quantitative analysis of cell apoptosis. (C) Bcl-2 and (D) Bax protein expression as assessed by western blot analysis. ${ }^{\mathrm{a}} \mathrm{P}<0.05$ vs. -Ang II; ${ }^{\mathrm{b}} \mathrm{P}<0.05$ vs. $+\mathrm{Ang}$ II; ${ }^{\mathrm{c}} \mathrm{P}<0.05$ vs. pc control + Ang II. Ang II, angiotensin II; -Ang II, untreated hPASMCs; +Ang II, Ang II-stimulated hPASMCs; control + Ang II, Ang II-stimulated hPASMCs not transfected with any plasmid; pc control + Ang II, Ang II-stimulated hPASMCs transfected with empty vector; pc IGFBP-3 + Ang II, Ang II-stimulated hPASMCs transfected with IGFBP-3 expression plasmid. 

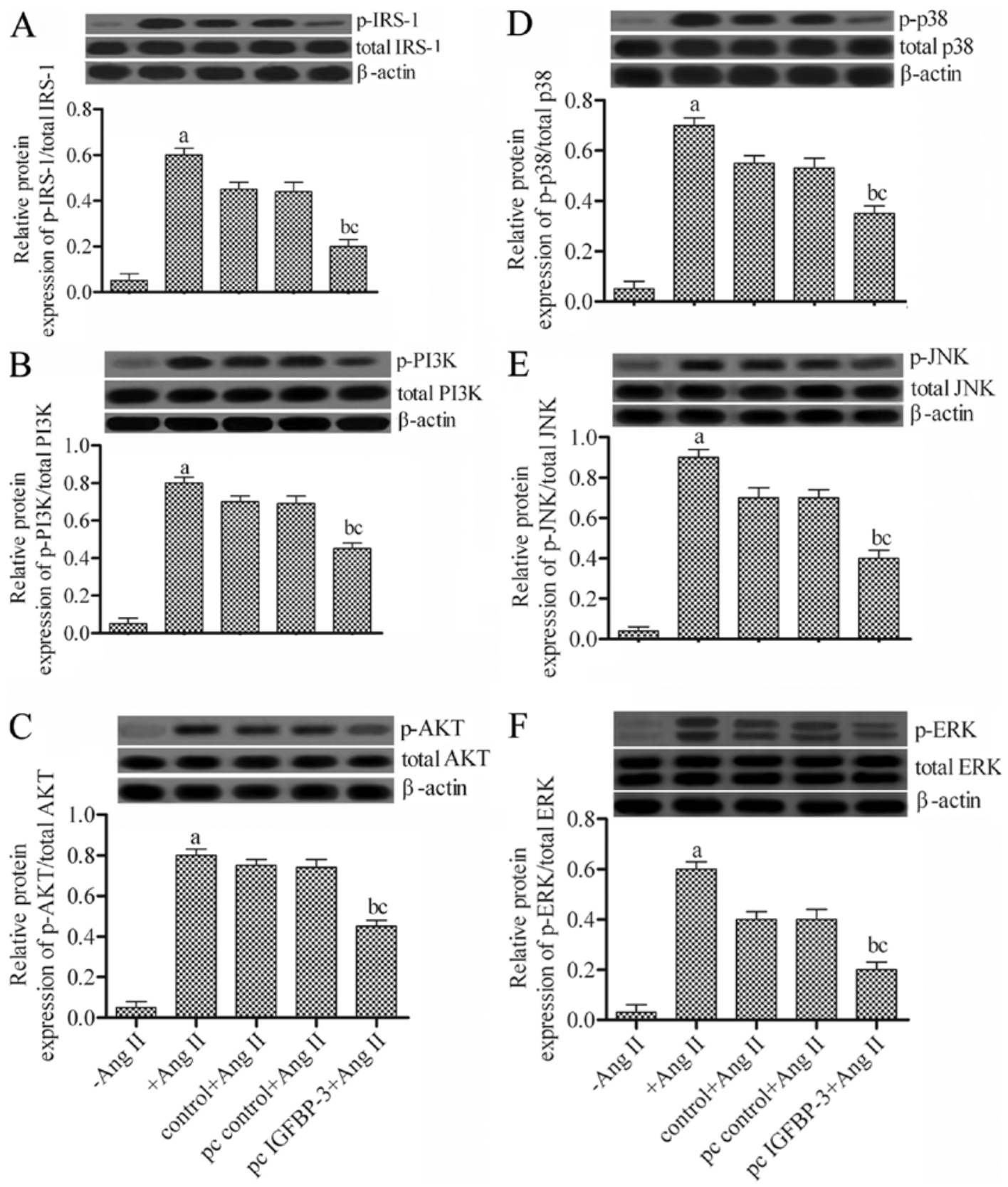

Figure 5. Effects of insulin-like growth factor binding protein-3 (IGFBP-3) overexpression on P13K-AKT and mitogen-activated protein kinase (MAPK) pathways in human pulmonary artery smooth muscle cells (hPASMCs). Protein expression levels of (A) insulin receptor substrate-1 (IRS-1), (B) PI3K, (C) AKT, (D) p38, (E) JNK and (F) ERK and their phosphorylated forms as detected by western blot analysis. ${ }^{\mathrm{a}} \mathrm{P}<0.05$ vs. $-\mathrm{Ang}$ II; ${ }^{\mathrm{b}} \mathrm{P}<0.05$ vs. + Ang II; ${ }^{\mathrm{c}} \mathrm{P}<0.05 \mathrm{vs}$. pc control + Ang II. Ang II, angiotensin II; -Ang II, untreated hPASMCs; +Ang II, Ang II-stimulated hPASMCs; control + Ang II, Ang II-stimulated hPASMCs not transfected with any plasmid; pc control + Ang II, Ang II-stimulated hPASMCs transfected with empty vector; pc IGFBP-3 + Ang II, Ang II-stimulated hPASMCs transfected with IGFBP-3 expression plasmid.

MSCs are multipotent progenitor cells and can be induced to differentiate into diverse cell lineages, such as cardiomyocytes, endothelial cells and VSMCs. Currently, MSCs are one of the cell types being used in clinical trials for the treatment of various diseases, including hematological diseases, organ transplantation, inflammatory diseases and autoimmune diseases (23). In addition, implanted MSCs promote tissue regeneration via the secretion of a variety of growth factors and cytokines (24). In this study, IGFBP-3 expression was detected by ELISA in hBM-MSCs (Fig. 1A), which is consistent with the results obtained that the relative intensity of IGFBP-3 is up to a value of 55\% in the supernatant of MSC culture (18). A recent study suggested that MSCs significantly ameliorated pulmonary arterial pressure and decreased right ventricle hypertrophy and pulmonary arteriole remodeling during the development of PAH in rats (25). In line with these findings, our data indicated that hBM-MSCs inhibit the proliferation of Ang II-stimulated hPASMCs following co-culture. We also demonstrated that DNA synthesis and the total protein levels in hPASMCs in co-culture were decreased (Fig. 2B and C).

Moreover, we assessed the pro-apoptotic effects of hBM-MSCs on hPASMCs (Fig. 4A and B) and the expression 
of Bcl-2 was also downregulated (Fig. 4C) and Bax expression was increased in the hPASMCs co-cultured with IGFBP-3modified hBM-MSCs (Fig. 4D), which may have contributed to the inhibition of the proliferation of hPASMCs. It has been demonstrated that endogenous IGFBP-3 facilitated the phosphorylation and nuclear export of orphan nuclear receptor Nur77 to the cytoplasm, where it exerts its apoptotic effect (26). IGFBP-3 also induces apoptosis and growth inhibition via IGF-1 independent mechanisms in various cell systems $(27,28)$. Of note, we found that IGFBP-3-modified hBM-MSCs exerted prominent inhibitory effects on the proliferation of hPASMCs. Our findings also indicated that IGFBP-3 was associated with cell apoptosis; however, further research is required to confirm that this molecular mechanism is consistent with that of previous research (29), which showed that IGFBP-3 blocked the type I IGF receptor (IGF1R)/PI3K/Akt survival signaling pathway, leading to cell apoptosis by sequestering IGF-1 away from the IGF1R.

In a previous study (30), the knockdown of IGFBP-3 was associated with only a subtle phenotype under control conditions. In additional, the idea that phenotype switching of PASMCs from a contractile to a synthetic phenotype plays a vital role in the progression of PAH is well established (31). The former type of VSMCs typically proliferate at a relatively low rate and produce a repertoire of smooth muscle-specific contractile proteins, such as $\alpha$-SM-actin. However, this synthetic phenotype is characterized by over-proliferation, the secretion of collagen, elastin and proteoglycans into the extracellular matrix $(32,33)$. Most noteworthy, our findings are the first to indicate, at least to the best of our knowledge, that IGFBP-3-modified hBM-MSCs significantly upregulate the level of $\alpha$-SM-actin and decrease OPN expression in hPASMCs compared to the controls following co-culture.

To explore the underlying mechanisms involved in the inhibition of cell proliferation and the promotion of apoptosis, and the phenotypic transformation hPASMCs by co-culture with IGFBP-3-modified hBM-MSCs, we examined IRS-1 expression and the expression of related proteins of the PI3K-AKT and MAPK signaling pathways, including p-P13K, p-AKT, p-p38, p-JNK and p-ERK. IRS-1 is a docking protein critical for mediating signals from IGF-1 and it has been reported that the overexpression of IGFBP-3 successfully restores the repressed levels of IRS-1 in primary human adipocytes (34). We also found that $I G F B P$-3-modified hBM-MSCs decreased the protein expression level of the p-IRS-1 in hPASMCs following co-culture. Our findings further proved that the protein expression of detected genes in hPASMCs was significantly downregulated following co-culture (Fig. 5). The PI3K/Akt signaling pathway has been shown to mediate VSMC proliferation, apoptosis and phenotypic transformation (35-37). Moreover, the MAPK pathway is also related to the proliferation and vascular remodeling of hPASMCs (38). In the present study, we demonstrated that the protein expression of $\mathrm{p}-\mathrm{PI} 3 \mathrm{~K}$, p-Akt, p-p38, p-JNK and p-ERK in hPASMCs was diminished compared to that of the controls following co-culture with IGFBP-3-modified hBM-MSCs, indicating that IGFBP-3 may mediate the proliferation, apoptosis and phenotypic transformation of hPASMCs via the PI3K/Akt and MAPK signaling pathways. It has been demonstrated that IGFBP-3 inhibits the IGF1R/PI3K/Akt survival signaling pathway by competitively binding IGF1R (29). In addition, IGF-1 rapidly induces the phosphorylation of IGF-1R followed by the activation of the AKT and MAPK signaling pathways in endometrial cancer lines (39). In addition, Ang II can promote IGF-1 mRNA expression in human umbilical artery smooth muscle cells (40). However, the precise mechanisms through which IGFBP-3 binds IGF1R, inhibits p-IRS-1 and then hinders the PI3K/Akt and MAPK signaling pathways in hPASMCs regulated by IGFBP-3-modified hBM-MSCs in the context of PAH remains unknown.

In conclusion, in this study, and to the best of our knowledge, we demonstrate for the first time that IGFBP-3-modified hBM-MSCs inhibit the proliferation and promote the apoptosis of hPASMCs, and also induced a phenotypic change to a contractile phenotype in hPASMCs. These effects may be exerted by acting upon the PI3K/Akt and MAPK signaling pathways. This study suggests that IGFBP-3-modified hBM-MSCs may be a promising therapeutic strategy for the treatment of $\mathrm{PAH}$. However, further research using animal model is required.

\section{References}

1. Ibrahim el-SH and Bajwa AA: Severe pulmonary arterial hypertension: Comprehensive evaluation by magnetic resonance imaging. Case Rep Radiol 2015: 946-920, 2015.

2. Nogueira-Ferreira R, Vitorino R, Ferreira R and HenriquesCoelho T: Exploring the monocrotaline animal model for the study of pulmonary arterial hypertension: A network approach. Pulm Pharmacol Ther 35: 8-16, 2015.

3. Perrin S, Chaumais MC, O'Connell C, Amar D, Savale L, Jaïs X, Montani D, Humbert M, Simonneau G and Sitbon O: New pharmacotherapy options for pulmonary arterial hypertension. Expert Opin Pharmacother 16: 2113-2131, 2015.

4. Weitzenblum E, Chaouat A, Canuet M and Kessler R: Pulmonary hypertension in chronic obstructive pulmonary disease and interstitial lung diseases. Semin Respir Crit Care Med 30: 458-470, 2009.

5. Xiao ZC and Liu YB: Treatment advance and tendency of pulmonary arterial hypertension. Clin Med Engineering 23: 257-260, 2016 (In Chinese).

6. Wang $\mathrm{CH}$ and An Y: Progress of stem cell treatment of pulmonary arterial hypertension. Chin J Clin Thorac Cardiovasc Surg 23: 294-298, 2016 (In Chinese).

7. Firth AL, Yao W, Ogawa A, Madani MM, Lin GY and Yuan JX: Multipotent mesenchymal progenitor cells are present in endarterectomized tissues from patients with chronic thromboembolic pulmonary hypertension. Am J Physiol Cell Physiol 298: C1217-C1225, 2010.

8. Takemiya K, Kai H, Yasukawa H, Tahara N, Kato S and Imaizumi T: Mesenchymal stem cell-based prostacyclin synthase gene therapy for pulmonary hypertension rats. Basic Res Cardiol 105: 409-417, 2010.

9. Bach LA: Insulin-like growth factor binding proteins - an update. Pediatr Endocrinol Rev 13: 521-530, 2015.

10. Kielczewski JL, Jarajapu YP, McFarland EL, Cai J, Afzal A, Li Calzi S, Chang KH, Lydic T, Shaw LC, Busik J, et al: Insulin-like growth factor binding protein-3 mediates vascular repair by enhancing nitric oxide generation. Circ Res 105: 897-905, 2009.

11. Moser DR, Lowe WL Jr, Dake BL, Booth BA, Boes M, Clemmons DR and Bar RS: Endothelial cells express insulin-like growth factor-binding proteins 2 to 6 . Mol Endocrinol 6: 1805$1814,1992$.

12. Johnson MA and Firth SM: IGFBP-3: A cell fate pivot in cancer and disease. Growth Horm IGF Res 24: 164-173, 2014.

13. Valentinis B, Bhala A, DeAngelis T, Baserga R and Cohen P: The human insulin-like growth factor (IGF) binding protein-3 inhibits the growth of fibroblasts with a targeted disruption of the IGF-I receptor gene. Mol Endocrinol 9: 361-367, 1995.

14. Lofqvist C, Chen J, Connor KM, Smith AC, Aderman CM, Liu N, Pintar JE, Ludwig T, Hellstrom A and Smith LE: IGFBP3 suppresses retinopathy through suppression of oxygen-induced vessel loss and promotion of vascular regrowth. Proc Natl Acad Sci USA 104: 10589-10594, 2007. 
15. Tajsic T and Morrell NW: Smooth muscle cell hypertrophy, proliferation, migration and apoptosis in pulmonary hypertension. Compr Physiol 1: 295-317, 2011.

16. Chen PK, Shi B, Long XP, Liu ZJ, Wang ZL and Wang DM: Effects of rat mesenchymal stem cells modified by CGRP on proliferation and phenotype transformation of vascular smooth muscle cells in vitro. Chin J Pathophysiology 29: 1777-1782, 2013 (In Chinese).

17. Su XY, Jiang XM and Chen SL: The expression profile of IGFBP family in pulmonary artery smooth muscle cells of rats with pulmonary hypertension. Zhonghua Linchuang Yishi Zazhi 9: 1143-1148, 2015 (In Chinese).

18. Schinköthe T, Bloch W and Schmidt A: In vitro secreting profile of human mesenchymal stem cells. Stem Cells Dev 17: 199-206, 2008.

19. Firth SM, Ganeshprasad U and Baxter RC: Structural determinants of ligand and cell surface binding of insulin-like growth factor-binding protein-3. J Biol Chem 273: 2631-2638, 1998.

20. Xia Y, Bhattacharyya A, Roszell EE, Sandig M and Mequanint K: The role of endothelial cell-bound Jagged1 in Notch3-induced human coronary artery smooth muscle cell differentiation. Biomaterials 33: 2462-2472, 2012.

21. Li Y, Liu G, Cai D, Pan B, Lin Y, Li X, Li S, Zhu L, Liao X and Wang $\mathrm{H}$ : H2S inhibition of chemical hypoxia-induced proliferation of HPASMCs is mediated by the upregulation of COX-2/PGI2. Int J Mol Med 33: 359-366, 2014

22. Liu Y, Tian HY, Yan XL, Fan FL, Wang WP, Han JL, Zhang JB, Ma Q, Meng Y and Wei F: Serotonin inhibits apoptosis of pulmonary artery smooth muscle cell by pERK1/2 and PDK through 5-HT1B receptors and 5-HT transporters. Cardiovase Pathol 22: 451-457, 2013.

23. Squillaro T, Peluso G and Galderisi U: Clinical trials with mesenchymal stem cells: An update. Cell Transplant 25: 829-848, 2016.

24. Baraniak PR and McDevitt TC: Stem cell paracrine actions and tissue regeneration. Regen Med 5: 121-143, 2010.

25. Chen JY, An R, Liu ZJ, Wang JJ, Chen SZ, Hong MM, Liu JH, Xiao MY and Chen YF: Therapeutic effects of mesenchymal stem cell-derived microvesicles on pulmonary arterial hypertension in rats. Acta Pharmacol Sin 35: 1121-1128, 2014.

26. Agostini-Dreyer A,Jetzt AE, Stires H and Cohick WS: Endogenous IGFBP-3 mediates intrinsic apoptosis through modulation of Nur77 phosphorylation and nuclear export. Endocrinology 156: 4141-4151, 2015.

27. Muzumdar RH, Ma X, Fishman S, Yang X, Atzmon G, Vuguin P, Einstein FH, Hwang D, Cohen P and Barzilai N: Central and opposing effects of IGF-I and IGF-binding protein-3 on systemic insulin action. Diabetes 55: 2788-2796, 2006.

28. Chan SS, Twigg SM, Firth SM and Baxter RC: Insulin-like growth factor binding protein-3 leads to insulin resistance in adipocytes. J Clin Endocrinol Metab 90: 6588-6595, 2005.

29. Chang RL, Lin JW, Hsieh DJ, Yeh YL, Shen CY, Day CH, Ho TJ, Viswanadha VP, Kuo WW and Huang CY: Long-term hypoxia exposure enhanced IGFBP-3 protein synthesis and secretion resulting in cell apoptosis in H9c2 myocardial cells. Growth Factors 33: 275-281, 2015.
30. Blouin MJ, Bazile M, Birman E, Zakikhani M, Florianova L, Aleynikova O, Powell DR and Pollak M: Germ line knockout of IGFBP-3 reveals influences of the gene on mammary gland neoplasia. Breast Cancer Res Treat 149: 577-585, 2015.

31. Schermuly RT, Ghofrani HA, Wilkins MR and Grimminger F: Mechanisms of disease: Pulmonary arterial hypertension. Nat Rev Cardiol 8: 443-455, 2011.

32. Mandegar M, Fung YC, Huang W, Remillard CV, Rubin LJ and Yuan JX: Cellular and molecular mechanisms of pulmonary vascular remodeling: Role in the development of pulmonary hypertension. Microvasc Res 68: 75-103, 2004.

33. Jeffery TK and Morrell NW: Molecular and cellular basis of pulmonary vascular remodeling in pulmonary hypertension. Prog Cardiovasc Dis 45: 173-202, 2002.

34. Mohanraj L, Kim HS, Li W, Cai Q, Kim KE, Shin HJ, Lee YJ, Lee WJ, Kim JH and Oh Y: IGFBP-3 inhibits cytokine-induced insulin resistance and early manifestations of atherosclerosis. PLoS One 8: e55084, 2013.

35. Wu J, Yu Z and Su D: BMP4 protects rat pulmonary arterial smooth muscle cells from apoptosis by PI3K/AKT/Smad1/5/8 signaling. Int J Mol Sci 15: 13738-13754, 2014.

36. Kiss T, Kovacs K, Komocsi A, Tornyos A, Zalan P, Sumegi B, Gallyas F Jr and Kovacs K: Novel mechanisms of sildenafil in pulmonary hypertension involving cytokines/chemokines, MAP kinases and Akt. PLoS One 9: e104890, 2014.

37. Garat CV, Crossno JT Jr, Sullivan TM, Reusch JE and Klemm DJ: Inhibition of phosphatidylinositol 3-kinase/Akt signaling attenuates hypoxia-induced pulmonary artery remodeling and suppresses CREB depletion in arterial smooth muscle cells. J Cardiovasc Pharmacol 62: 539-548, 2013.

38. Biasin V, Chwalek K, Wilhelm J, Best J, Marsh LM, Ghanim B, Klepetko W, Fink L, Schermuly RT, Weissmann N, et al: Endothelin-1 driven proliferation of pulmonary arterial smooth muscle cells is c-fos dependent. Int J Biochem Cell Biol 54: 137-148, 2014.

39. Mendivil A, Zhou C, Cantrell LA, Gehrig PA, Malloy KM, Blok LJ, Burger CW and Bae-Jump VL: AMG 479, a novel IGF-1-R antibody, inhibits endometrial cancer cell proliferation through disruption of the PI3K/Akt and MAPK pathways. Reprod Sci 18: 832-841, 2011.

40. Zha Z, Zhang QH, Jiang ZX, Chen L, Lin H and Liang XM: Effect of angiotensin II on pregnancy-associated plasma protein $\mathrm{A}$ and insulin-like growth factor 1 gene expression in human umbilical artery smooth muscle cells. Nan Fang Yi Ke Da Xue Xue Bao 29: 195-198, 2009 (In Chinese). 